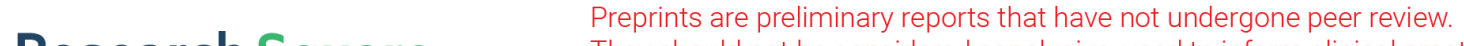 They should not be considered conclusive, used to inform clinical practice, or referenced by the media as validated information. \\ Regulation of Follicular Atresia by WIP1-Mediated Apoptosis and Autophagy
}

\section{Su Zhou}

Huazhong University of Science and Technology

\section{Yueyue Xi}

Huazhong University of Science and Technology

\section{Yingying Chen}

Huazhong University of Science and Technology

\section{Wei Yan}

Huazhong University of Science and Technology

\section{Meng Wu}

Huazhong University of Science and Technology

\section{Milu Li}

Huazhong University of Science and Technology

\section{Tong Wu}

Huazhong University of Science and Technology

\section{Jinjin Zhang}

Huazhong University of Science and Technology

\section{Wei Shen}

Huazhong University of Science and Technology

\section{Aiyue Luo}

Huazhong University of Science and Technology

Shixuan Wang ( $\nabla$ shixuanwang@tjh.tjmu.edu.cn )

Huazhong University of Science and Technology

\section{Research}

Keywords: WIP1, follicular atresia, granulosa cell, apoptosis, autophagy, ovarian aging

Posted Date: August 20th, 2021

DOl: https://doi.org/10.21203/rs.3.rs-826867/v1

License: (1) This work is licensed under a Creative Commons Attribution 4.0 International License. 

2 Su Zhou, Yueyue Xi, Yingying Chen, Wei Yan, Meng Wu, Milu Li, Tong Wu, Jinjin Zhang, Wei 3 Shen, Aiyue Luo, Shixuan Wang*.

4

Department of Obstetrics and Gynecology, Tongji Hospital Affiliated to Tongji Medical College, Huazhong University of Science and Technology. 1095 Jiefang Ave, Wuhan 430030, China

\section{*Correspondence:}

Shixuan Wang

Email: shixuanwang@tjh.tjmu.edu.cn (S. W.)

Keywords: WIP1, follicular atresia, granulosa cell, apoptosis, autophagy, ovarian aging

\section{Abstract}

Female endocrine homeostasis and reproductive success depend on the number and quality of follicles. The follicle is the basic functional unit within mammalian ovaries. Excessive follicular atresia is responsible for the accelerated ovarian aging process. Therefore, exploring the molecular mechanism of follicle development and atresia is essential for protecting ovarian function. In this study, we interrogate the striking correlation between follicular atresia and wild-type p53-induced phosphatase 1 (WIP1) expression in mouse ovaries to understand how WIP1 phosphatase activity regulates follicle development. WIP1 is mainly expressed in granulosa cells of healthy growing follicles, and atretic follicles exhibit significantly weaker WIP1 expression compared with the healthy ones. Our in vivo study indicates that inhibition of WIP1 phosphatase activity causes 
endocrine disorder, fertility decline and decreased ovarian reserve by triggering excessive follicular atresia through promoting autophagy and apoptosis. By in vitro follicle culture, we determine that inhibiting the WIP1 activity impairs the follicle development, causing more follicular atresia and decreased oocyte quality. Besides, downregulating WIP1 expression in granulosa cells in vitro also promotes apoptosis and autophagy via WIP1-p53 and WIP1-mTOR signal pathway. Our findings from the in vitro and in vivo experiments revealed that appropriate Wip1 expression is required for follicle development. Downregulation of WIP1 expression accelerates follicle atresia via WIP1-p53 and WIP1-mTOR signal pathway related apoptosis and autophagy. It is speculated that moderate up-regulation of WIP1 expression may help delaying the decline of ovarian reserve.

\section{Introduction}

The age-related decline in ovarian function is the major challenge in women's reproductive health.

The decline in endocrine function or reproductivity is closely related to the quality and quantity of follicles. The decline of the ovarian follicular reserve is nonlinear and seems to accelerate with age $[1,2]$. The mechanisms of the accelerated depletion of follicles in later reproductive ages remain to be elucidated. Exploring the mechanism may help developing targeted treatment strategies to slow down the depletion of ovarian follicles and delay ovarian aging. Moreover, menopause related disease such as cardiovascular disease [3], osteoporosis [4], neurodegenerative diseases [5], etc. could also be improved.

The healthy follicular development and oocyte maturation are the prerequisite for endocrine function and obtaining high-quality oocytes. Follicular atresia is also a survival of the fittest physiological phenomenon that occurs throughout the ovarian life. Abnormalities in follicular development and atresia will inevitably lead to abnormal ovarian function [6,7]. Both external and 
internal factors induce cell DNA damage. The DNA double-strand breaks can alter genetic integrity and cause deleterious damage to cells. Previous studies have demonstrated that decreased oocyte quality in older individuals is closely related to increased DNA damage [8]. DNA damage in granulosa cells also accumulates with age [9]. If the DNA damage cannot be repaired, cells undergo programmed cell death or senescence to avoid severe mutagenic consequences [10].

The size of the follicle pool and the depletion rate of primordial follicles determine the ovarian functional lifespan [11]. Apoptosis is regarded as the main mechanism of the follicular atresia, whereas the follicle destiny is determined by the balance between proapoptotic and prosurvival molecules [7,11-13] Recently, a few studies indicate that autophagy also participates in the regulation of follicle development $[14,15]$. However, the molecular mechanisms that regulate follicular development and the ovarian aging still need to be explored.

Wip1, coded by Ppm1d, is a Ser/Thr protein phosphatase of PP2C family. A number of proteins, regulating the DNA damage response and cellular checkpoint pathways, could be dephosphorylated by Wip1 [16]. Previous studies suggested that Wip1 was highly expressed in various tumor tissues [17-22]. In recent years, more and more researches pay attention to the regulatory role of WIP1 in aging [23-26]. The islet B cells and neural stem cells of aged mice exhibit lower expression of Wip1 compared with the younger ones[27,28]. The lack of Wip1 phosphatase leads to the over-activation of p38MAPK pathway, promoting the enhanced expression of aging related genes, such as INK4a and ARF, and accelerates aging [27]. Upregulation of WIP1 expression in mice using genetic engineering technology can increase the number of neural stem/progenitor cells and induce a higher degree of neuronal differentiation. In addition, augmenting WIP1 of in aged animals can promote the formation of neurons and restore olfactory function [28]. Our recent study also indicated that the ovaries of aged mice exhibited significantly 
1 lower WIP1 expression than the younger ones[29]. However, whether WIP1 is involved in the ovarian aging process need to be explored.

Deletion of Wipl has been proved to cause abnormal spermatogenesis and sperm maturation in the mice, resulting in declined fertility [30-32]. Leem J et al. reported that WIP1 suppresses DNA repair in oocytes [33]. Our recent study has revealed that WIP1 regulates the primordial follicle development in neonatal mouse ovaries, indicating its potential role in regulating ovarian reserve [29]. While, the regulatory role of WIP1 in ovarian function remains to be further clarified. This study was aimed to explore the specific role and mechanism of WIP1 phosphatase in regulating ovarian follicular development in adult mice. Our objective was to investigate the possible role of WIP1 and its related signal pathways in ovaries to provide guidance for finding strategies to delay ovarian aging.

\section{Materials and methods}

Postnatal day (PND) 14 and PND21 C57BL/6j female mice, purchased from the Center for Disease and Prevention of Hubei Province (Wuhan, China), were sacrificed for primary cell or organ culture. Thirty-six female mice (C57BL/6j, 6 weeks old, purchased from Beijing Huafukang BioTechnology Co., Ltd.) were fed freely for one week to acclimate to the environment. All animals were fed with standard chow and water ad libitum under appropriate temperature and humidity. All in vivo experiments were carried out in accordance with the approved guidelines by the ethics committee of Tongji Hospital, Tongji Medical College, Huazhong University of Science and Technology.

The mice were divided into three groups (with 12 female C57BL/6J mice in each group): Vehicle group (Veh), $7.5 \mathrm{mg} \mathrm{kg}^{-1}$ GSK2830371 (MedChemExpress USA, HY-15832) group (GSK7.5), $15 \mathrm{mg} \mathrm{kg}^{-1}$ GSK group (GSK-15). Mice were respectively administered with Vehicle or GSK 
12830371 twice a day (9:00 and 18:00) by oral gavage for 14 consecutive days. The following pure

solvents (2\% DMSO, 30\% PEG300, 5\% Tween 80, dd $\mathrm{H}_{2} \mathrm{O}$ ) were added to the GSK2830371 in turn. Mice were sacrificed to extract samples 2 hours after the last administration.

\section{Immunohistochemistry}

The collected mouse ovaries were embedded in paraffin, and serially sectioned $(5 \mu \mathrm{m})$. As previously described [34], the ovary sections were incubated with primary antibody (anti-WIP1 (F10) (sc-376257, 1:200, Santa Cruz Biotechnology, CA, USA; LC3B Antibody, 1:100, A19665, ABclonal, Wuhan, China; SQSTM1Antibody, 1:100, A11483, ABclonal, Wuhan, China) overnight at $4^{\circ} \mathrm{C}$. The next day the ovary sections were incubated with a secondary antibody (HRP-labeled goat Anti-rabbit/mouse IgG (H+L), Servicebio, GB23204, 1:200) for 60 minutes at $37^{\circ} \mathrm{C}$. Subsequently the ovary sections were visualized with DAB-HRP chromogenic agent (DAKO, K5007) at room temperature for 20 seconds. The negative control was sections treated with AffiniPure mouse $\operatorname{IgG}(\mathrm{H}+\mathrm{L})(2 \mu \mathrm{g} / \mathrm{mL}$, BA1046, Boster, Wuhan, China) or normal rabbit IgG (2 $\mu \mathrm{g} / \mathrm{ml}, \mathrm{BA} 1044$, BOSTER). Microscopy and the images were performed by microscope (version 1.8.1, Olympus) with the cellSens Dimension software. Image Pro Plus software was used to evaluate the relative expression.

\section{Estrous cycle monitoring and mating test}

After two weeks treatment, the estrous cycles were monitored at the age of 9 weeks. The vaginal smears were tested at 9:00 am. every day for consecutive two weeks as previously described [35]. Mating test was carried out after two weeks' estrous cycle monitoring as showed in Fig.1A. The pregnant cohabited female mice and their litter size were evaluated.

\section{Serum AMH level by ELISA}

Blood samples were collected after two weeks' GSK2830371 treatment. The enzyme-linked 
immune sorbent assay (ELISA) Kit (Bioss) was used to detect the serum AMH levels according to the manufacturer's instructions.

\section{Follicle counting}

The ovary sections harvest after two weeks' GSK2830371 treatment were stained with hematoxylin and eosin for follicle counting as previously described [35]. Briefly, every four slide was observed under the microscope and only follicles with oocytes were counted. Two persons counted independently and the counting results were analyzed together.

\section{Apoptosis test}

The In-Situ Cell Death Detection Kit (Roche, Basel, Switzerland) was applied for analysing cell apoptosis. As the manufacturer's instructions suggested, the ovarian sections were incubated with terminal deoxynucleotidyl transferase-mediated nick-end labeling (TUNEL) reaction mixture at $37^{\circ} \mathrm{C}$ for 60 minites. The images with the green fluorescence of the apoptotic cells were acquired with the cellSens Dimension software and microscope (version 1.8.1, Olympus). The TUNEL positive cells were calculated by Image-Pro Plus.

\section{Western blot}

Total protein was isolated from mouse ovaries or cultured cells for Western Blot experiments as described previously [34]. The primary antibody: Anti-WIP1 (F-10) (sc-376257, 1:200, Santa Cruz Biotechnology, CA, USA), Anti-gamma H2AX (phospho S139) (ab81299, Abcam, Cambridge, MA, USA), Phospho-ATM (Ser1981) (AF8225, Affinity Bioscience), Phospho-p53 (Ser15) (\#9284, Cell Signaling Technology, Beverly, MA, United States ), Bax (ab32503, Abcam, Cambridge, MA, USA), Bcl-2 (ab182858, Abcam, Cambridge, MA, USA), Cleaved-Caspase3 (ab49822, Abcam, Cambridge, MA, USA), Phospho-mTOR-S2448 (AP0115, ABclonal, Wuhan, China), mTOR 
1 (GB11405, Servicebio, Wuhan, China), WIP1 Polyclonal Antibody (A6204, ABclonal, Wuhan,

China); LC3B Antibody (A19665, ABclonal, Wuhan, China); SQSTM1Antibody (1:100, A11483, ABclonal, Wuhan, China); GAPDH (ANT012, AntGene). Image Lab (Java image processing software, BioRad, Hercules, CA, USA) was used to quantified the integrated light intensity. GAPDH expression was used to verify equal loading.

\section{Follicle Isolation and Follicle Encapsulation}

The methods of follicle isolation and culture were described previously [36,37]. Briefly, the PND14 mouse ovaries were removed and the follicles were mechanically dissected with syringe needles. Intact follicles (diameter: 120-140 $\mu \mathrm{m}$ ) with a spherical oocyte in the central were selected for in vitro culture. The sodium alginate solution (Sigma-Aldrich, St.Louis, MO. A2033; 2\% (w/v)) and $50 \mathrm{mM} \mathrm{CaCl}_{2}+140 \mathrm{mM} \mathrm{NaCl}$ solution were used to encapsulate follicles as previous described [36].

The follicle culture medium was $\alpha$-minimal essential medium (Life technologies corporation, Gaithersburg, MD) supplemented with 10\% FBS (10099; Gibco, Grand Island, NY), 100 IU/ml of penicillin, $100 \mu \mathrm{g} / \mathrm{ml}$ of streptomycin, Insulin Transferrin Selenium $(5 \mu \mathrm{g} / \mathrm{mL}$ of insulin, $5 \mu \mathrm{g} / \mathrm{mL}$ of transferrin, and $5 \mathrm{ng} / \mathrm{mL}$ of selenium) (Sigma-Aldrich, St. Louis, MO) and $10 \mathrm{mIU} / \mathrm{ml}$ recombinant mouse follicle-stimulating hormone (rFSH: 8576-FS; R\&D systems, Minneapolis, MN). Follicles were cultured at $37^{\circ} \mathrm{C}$ in $95 \%$ air $-5 \% \mathrm{CO} 2$ for 6 days.

\section{In vitro Oocyte Maturation}

After 6 days culture, the remaining alginate around the follciles is degraded by alginate-lyase at $37^{\circ} \mathrm{C}$ for $20-25$ minutes. For in vitro oocyte maturation (IVM), the cultured follicles were transferred to the IVM medium, consisting of $\alpha \mathrm{MEM}, 10 \% \mathrm{FCS}, 1.5 \mathrm{IU} / \mathrm{mL}$ of hCG, and $5 \mathrm{ng} / \mathrm{mL}$ of epidermal growth factor (EGF) and transfered to $\mathrm{CO}_{2}$ incubator for $15-16$ hours. Then the 
cumulus complex was incubated with hyaluronidase solution $(0.1 \mathrm{mg} / \mathrm{mL})$ to remove the cumulus granulosa cells with micropipette tip pipetting several times. The oocyte stages are defined as follows: Germinal vesicle (GV) stage, the oocyte did not resume meiosis in response to hCG exposure, and the nucleus of the oocyte persists; Germinal vesicle breakdown (GVBD), the nuclear membrane of oocytes disappeare, but no polar body is observed; Metaphase-II arrested oocyte (MII), the oocyte resumed meiosis, and a polar body could be observed under a dissecting microscope; Degenerated, the oocyte is fragmented several pieces [37].

\section{Immunofluorescence}

Deparaffinized and rehydrated follicle sections were blocked with $10 \%$ normal goat serum for 60 minutes, then incubated with Ki67 antibody (1:200, GB111141, Servicebio, Wuhan, China) overnight at $4^{\circ} \mathrm{C}$. The next day the ovary sections were incubated with secondary antibodies $(1: 200$, Alexa Fluo ${ }^{\circledR} 488$ Donkey anti Rabbit IgG $(\mathrm{H}+\mathrm{L})$, AntGene, ANT024) for $60 \mathrm{~min}$ at $37^{\circ} \mathrm{C}$. Normal rabbit IgG was used as the negative control. Images were acquired with microscope (version 1.8.1, Olympus) and the cellSens Dimension software.

\section{Primary granulosa cell culture and siRNA interference}

Primary granulosa cells were harvested as previously described [38]. After 48 hours culture of the granulosa cells, the Ppm ld-siRNA and negative control siRNA were transfected using the lipofectamine 3000 reagent (Invitrogen, USA) according to the manufacturer's instructions. Mouse Ppmld-siRNA and control siRNA were purchased from Riobio, Inc. (Guangzhou, China). Mouse Ppm1d-siRNA: si-m-Ppm1d_003 (siG151016012651), the target gene sequence:

\section{GCACCGACGAAATGGCTTA.}

\section{Cell proliferation detection by EdU}

The primary granulosa cell proliferation was analyzed using the Cell-Light EdU Apollo643 In Vitro 
1 Kit (C10310-2, RiboBio, Guangzhou, China) according to instructions provided by the manufacturer. The images were acquired with the cellSens Dimension software. The EdU positive cells were calculated by Image-Pro Plus.

\section{Cell apoptosis detection}

FITC-Annexin V Apoptosis Detection Kit I (BD Pharmingen ${ }^{\mathrm{TM}}$ ) was applied to detect cell apoptosis. The primary granulosa cells were treated with Si-RNA for 48 hours. Then harvested cells were incubated with Annexin V-PI for 15 minutes. The population of cells labeled by FITCAnnexin V or PI was analyzed by flow cytometer (BD Pharmingen, San Diego, CA, USA).

\section{Statistical Analysis}

All data are expressed as Means (SD) unless noted otherwise. Statistical comparisons were performed using unpaired Student's t tests, one-way ANOVA or Chi-square test. Statistical analysis was performed by SPSS (version 13.0). The significance level was set at $p<0.05$.

\section{Results}

\section{WIP1 expression in Follicles of Different Stages in Mouse Ovaries.}

The IHC analysis in 6-week-old mouse ovaries showed that WIP1 is mainly expressed in oocytes and granulosa cells, and the stromal cells exhibited weaker WIP1 expression. WIP1 was highly expressed in the granulosa cells of healthy follicles, while WIP1 expression was significantly downregulated in the granulosa cells of atretic follicles (Fig.1A). The relative WIP1 expression levels in follicles of healthy growing follicles (secondary follicles and antral follicles) and atretic follicles were showed in Figure1B, the atretic follicles showed significant weaker WIP1 expression compared with the healthy growing follicles.

\section{Inhibition of WIP1 Activity Led to Endocrine Disorder and Declined Reproductivity.}

To confirm the role of WIP1 in ovarian function and its mechanism, the in vivo WIP1 inhibitor 
1 (GSK2830371) intervention experiment was carried out as showed in Fig.2A. The body weight of

mice in the three groups showed no significant difference (Fig.2B). In the GSK-15 group, significantly fewer mice (20\%) showed regular estrous cycles compared with Veh group $(p<0.05)$ (Fig.2C). We observed that fewer mice became pregnant in the GSK groups than the Veh group ( $p<$ 0.05) (Fig.2D). The average litter size of total mated mice in GSK groups was lower than that of the Veh group with no significant difference $(p>0.05)$ (Fig.2E).

\section{Inhibition of WIP1 Activity Led to Declined Ovarian Reserve in Mice.}

The ovary index (ratio of ovary weight to body weight) also showed no significant difference among the three groups (Fig.2F). The serum AMH level decreased significantly in the GSK-15 group compared with Veh group, while the GSK-7.5group showed no significant difference (Fig.2G). The serial sections of ovaries were stained with H\&E for evaluation of ovarian reserve and follicular development (Fig.2H). The GSK2830371 treated mice exhibit fewer healthy follicles and more atretic follicles. The number of secondary follicles and antral follicles in the GSK-7.5 group was significantly lower than that in the Veh group $(* P<0.05)$. In the GSK-15 group, the number of primordial follicles was significantly lower than that in Veh group $(* P<0.05)$, while the number of atretic follicles was significantly higher than that in Veh group $(* P<0.05)$ (Fig.2I). The total healthy follicles in both intervention groups decreased significantly compared with the Veh group $(* P<0.05)$. According to the follicle counting results, we calculated the proportion of primordial, growing (containing primary, secondary and antral follicles) and atretic follicles (Fig.2I). The primordial follicle proportion decreased and the atretic follicles increased significantly in the GSK-15 group compared with the Veh group $\left({ }^{*} P<0.05\right)$, and the percentage of growing follicles in the total number of follicles increased slightly (Fig.2J). According to the statistics of follicle counting, we found that inhibiting the activity of WIP1 phosphatase in mice could 
accelerate the depletion of the primordial follicle pool and the occurrence of follicular atresia.

\section{The WIP1 related Mitochondrial Apoptosis and Autophagy Promoted the Follicle Atresia.}

Current studies have revealed that granulosa cell apoptosis is widely considered as the underlying mechanism of follicular atresia $[7,12]$. WIP1 has also been proved to be involved in regulating the cell apoptosis $[39,40]$. The TUNEL results demonstrated that the more apoptotic granulosa cells appeared in GSK group ovaries than that in the Veh group (Fig.3A-B). The key proteins involved in the DNA damage and the apoptosis process were detected by Western blot, and the results showed a significant increase in $\gamma$-H2AX, p-ATM (S1981), p-p53 (S15), BAX and cleaved-Caspase3 expression in ovaries of GSK groups compared with the Veh group (Fig.3C-D), while the antiapoptosis protein Bcl-2 decreased significantly in the GSK groups.

Autophagy, another important form of cell death, has also been proved to be involved in follicular atresia [14]. Autophagy is also highly regulated by a range of protein phosphorylation and dephosphorylation $[41,42]$. WIP1, as a phosphatase, has also been reported be involved in regulating the autophagy process in atherosclerosis $[43,44]$. mTOR signal pathway is a key regulator of autophagy [45]. To explore the autophagy in the mouse ovaries, we detected LC3B by IHC, and the results indicated that the LC3B expression, mainly in follicle granulosa cells, was significant stronger in the follicles of GSK groups compared with the Veh group (Fig.4A-B), indicating the autophagy activation in the follicles is one of the causes of follicular atresia. While the SQSTM1 expression in follicle granulosa cells was relatively weaker. The SQSTM1 was highly expressed in ovarian stroma (Fig.4C). The total SQSTM1 expression in GSK groups was significantly higher than that in Veh group (Fig.4D), which indicated that the autophagy in the ovarian stroma was inhibited by the GSK treatment. Western blot results showed the activation of mTOR signal pathway in GSK groups and a slight increase of SQSTM1 and LC3B II in the GSK- 
17.5 group (Fig.4E-F). Collectively, the levels of autophagy were not consistent in different parts of ovaries.

\section{Inhibition of WIP1 Activity Limited the Follicle Growth and Impaired the Oocyte Quality in} vitro.

To further clarify the influence of WIP1 on follicular development and atresia, preantral follicles were cultured with WIP1 inhibitor GSK2830371 $(0,0.1 \mu \mathrm{M}, 1.0 \mu \mathrm{M}, 5.0 \mu \mathrm{M}, 10.0 \mu \mathrm{M})$ for 6 days. To explore the effect of WIP1 on preantral follicle growth, the follicle morphology was observed and recorded daily (Fig.5A). The analysis of follicular diameter suggested that GSK2830371 intervention $(1.0 \mu \mathrm{M}, 5.0 \mu \mathrm{M}, 10.0 \mu \mathrm{M})$ could significantly inhibit follicular growth (Fig.5B). The follicles cultured in the absence of GSK2830371 or at low concentration of GSK2830371 $(0.1 \mu \mathrm{M})$ kept growing and exhibited their maximal volume at the 6th day of culture. The cell proliferation was detected by Ki67 staining, and follicles in the GSK group showed a reduction in Ki67 fluorescence intensity (Fig.5C). The TUNEL assay was used to compare the apoptosis in follicles after 4 days culture. The relative quantitative of TUNEL-positive GCs (staining with green) of the GSK group follicles significantly increased compared with the control group (Fig.5D). After 6 days' culture, the oocyte nuclear maturity was assessed to reflect the oocyte quality (Fig.5E). As shown in Fig.5F, the proportion of degenerated oocytes significantly increased in the GSK group, and the oocytes in GVBD and MII phase declined compared with the control group (P $<0.05)$. The results indicate inhibition of phosphatase activity can limit follicle growth and oocyte maturation, and the decreased proliferation and increased apoptosis granulosa cells induced by WIP1 inhibitor attribute to it.

\section{Downregulation of WIP1 Promoted the Mouse Granulosa Cell Autophagy and Apoptosis.}

The follicle development is closely related to the granulosa cells viability. To verify the relationship 
between WIP1 expression and granulosa cell proliferation and apoptosis, primary mouse granulosa cells were isolated and cultured for $24 \mathrm{~h}$ and then transfected with Si-ppm1d-RNA. The morphology of granulosa cells was observed and recorded (Fig.6A). After transfection 48 h, EdU immunofluorescent staining results showed that cell proliferation decreased in the Si-ppm1d group compared with the Si-Nc group (Fig.6B). Cell apoptosis increased significantly in the Si-ppm1d group compared with the Si-Nc group, as evidenced by Fluorescence activated Cell Sorting (FACS) apoptosis detection (Fig.6C) and the TUNEL staining (Fig.6D). Western blot results indicate that the mTOR regulated autophagy and the mitochondrial apoptosis pathway were both activated

$$
\text { (Fig.6E-F). }
$$

\section{Discussion}

This present study demonstrates that the WIP1 expression of atretic follicles is significantly lower than that of the healthy follicles. Our in vivo and in vitro experiments suggest that WIP1 related abnormal apoptosis and autophagy in granulosa cells lead to follicular atresia, also impairing the oocyte quality. WIP1 plays an essential role in regulating the ovarian reserve and ovarian function.

Follicular development and atresia are the key factors affecting ovarian function and ovarian life span. Follicular development and follicular atresia are finely regulated by a network of intracellular and extracellular signaling molecules [12]. Spatial and temporal regulation of protein phosphorylation and dephosphorylation plays a key role in controlling various signal pathways [46]. The phosphatase activity plays an important role in the regulation of follicular development. PTEN as a phosphatase, has been proved to be necessary in the maintenance of primordial follicle pool. PTEN phosphatase acts as the upstream of PI3K-AKT pathway, and helps to maintain the quiescence of primordial follicles. Deletion of PTEN causes overactivation of primordial follicles [47]. Our present study verified that WIP1 phosphatase also participates in the regulation of 
follicular development.

WIP1, as a phosphatase, regulates the DNA damage response, apoptosis [48-50], autophagy process $[43,44]$. Recently, more studies focus on its role in the aging process [23-26], except for its role in the tumorigenesis. One important hallmark of aging is the accumulation of DNA damage [51]. The integrity and stability of DNA are continually disturbed by exogenous and endogenous threats [52]. A sophisticated network of DNA repair mechanisms has evolved in organisms.

Emerging research indicates the importance of WIP1 in the regulation of stress-induced DNA damage and the relevant networks $[48,53-55]$. Our recent data have demonstrated that the expression of Wip1 in mouse ovaries decreased with age [29], which is consistent with the trends in other tissues $[23,27,28]$. The DNA damage accumulation and the decreased ability of DNA damage repair leads to more apoptosis in ovarian cells and follicle atresia. BRCA proteins (BRCA1, BRCA2) participated in the transcriptional regulation of DNA damage as well as the DNA repair process [56]. Clinical data and animal experiments have indicated that defects in BRCA1-related DNA double-strand break repair could induce ovarian reserve decline, accelerating ovarian aging [57]. The Brca2-deficient mice showed abnormal follicular development and oocyte quality impairment, because cellular DNA damage accumulated [58]. In our current study, WIP1 expression has been demonstrated to be associated with follicular development. Atretic follicles exhibited lower WIP1 expression compared with the healthy ones.

Previous studies have demonstrated apoptosis is the main mechanism mediating the follicular atresia [7,12]. Recently studies indicated that autophagy is also closely linked to follicular development [14]. Our data extend the previously identified role of apoptosis and autophagy in follicular development, and emphasize the process is also regulated by WIP1. Some examples of WIP1 targets include p53, ATM and $\gamma-\mathrm{H} 2 \mathrm{AX}$, involving in cell cycle arrest, apoptosis, DNA repair 
etc. [49]. Persistent ATM activation leads to impaired DNA repair and cell survival. Wip1 could dampen the stress response through dephosphorylating p53 or ATM [59]. The study by Dmitry V. Bulavin's team demonstrated that Wip1 deficiency or downregulation could activate autophagy through the ATM-mTOR signaling pathway, suggesting modulating WIP1-related autophagy could help preventing atherosclerosis $[43,44]$. A recent study also demonstrated that Wip1 related p53 pathway controls the cell fate through regulating autophagy and apoptosis [60]. Our current study demonstrated that inhibiting Wip1 activity led to enhanced apoptosis and autophagy in ovarian granulosa cells. Consistent with previous studies, the p53 pathway activation in granulosa cells induced by WIP inhibition contributed to both cell death phenotypes [60]. The mTOR signal pathway, the negative regulator of autophagy, was significantly inhibited after downregulating WIP1, causing excessive autophagy in granulosa cells. The abnormal cell death eventually leads to follicular atresia and decreased ovarian reserve.

Our previous study has already demonstrated that the WIP1 expression in mouse ovaries declined with age [29]. The WIP1 phosphatase activity decline is closely associated with impaired follicular development, as well as the ovarian aging. The age-dependent WIP1 downregulation accelerated the atresia of growing follicles, indirectly accelerating the depletion of the primal follicle pool. Similar to our study, Wip1 has also been proved to play a key role in several physiological processes such as neurogenesis and organismal aging [27,28]. Another study also shows that Wip1 is highly expressed in haematopoietic stem cell (HSC) but decreases with age, and Wip1-deficient mice exhibited multifaceted HSC aging phenotypes, depending on the distinct effects of p53 and mTORC1 pathways governed by Wip1 [61]. Wip1 has also been demonstrated to play a key role in preventing aging-related B-cell development dysfunction [62]. Collectively, low Wip1 expression or Wip1 deficiency is related to organismal aging, which is consistent with the 
results in the current study.

Previous studies have demonstrated that Wipl deficiency affects spermatogenesis and sperm maturation, resulting in reduced fertility in males [30-32]. While Wip1 in female reproductivity has not been studied extensively. The role of WIP1 in mouse oocytes has been studied in vitro, suggesting that WIP1 suppresses DNA repair in oocytes [33]. Inhibition of WIP1 phosphatase could improve the ability of oocytes to repair DNA damage [33]. Our current study mainly explored the role of WIP1 in granulosa cells. The role of Wip1 in granulosa cells seems different from its role in oocytes. Our study indicated that inhibition of Wip1 phosphatase may impair DNA damage repair ability and promote granulosa cell apoptosis through p53 signal pathway, resulting in more follicle atresia and declined ovarian reserve.

There are some limitations of our current study: First, intragastric drug administration used in the animal study, may affect the whole body not only the ovary. The effect on the ovary may be an indirect effect of systemic reactions. Although, in vitro studies in follicles and granulosa cells have also demonstrated the role of Wip1 in ovarian cells, it will be better if conditioned knockout mice (granulosa cell Wipl deficient) were used. Second, we only demonstrated low expression of Wip1 is detrimental to ovarian function. In the future, we still need to demonstrate whether augmenting Wip1 expression by genomic or pharmacologic methods in aged animals could improve ovarian reserve and delay ovarian aging.

\section{Conclusion}

In summary, our findings from the in vitro and in vivo experiments revealed that appropriate WIP1 activity is required for follicle development. Inhibition of WIP1 activity accelerates follicular atresia via WIP1-p53 and WIP1-mTOR signal pathway related apoptosis and autophagy in granulosa cells. 


\section{Acknowledgements}

3 This research was supported by the grant from the National Natural Science Foundation of China. The

4 important contributions of our graduate students and colleagues in this research are gratefully acknowledged.

5

6

\section{Authors' contributions}

Su Zhou: designed and performed the research, analyzed the data, wrote the paper; Yueyue Xi and Yingying Chen: executed the in vivo and in vitro experiments; Wei Yan, Meng Wu and Tong Wu: contributed essential reagents or tools; Wei Shen and Yan Li and Ai-yue Luo: critical discussion; Shixuan Wang: research design, critical discussion, review and editing.

\section{Funding}

This work was supported by the grant from National Natural Science Foundation of China, Grant/Award Number: 81873824 and 81701438.

\section{Availability of data and materials}

The data that support the findings of this study are included in this article. The original data underlying this article will be shared on reasonable request to the corresponding author.

\section{Declarations}

\section{Ethics approval and consent to participate}

All animal procedures and protocols were approved by the ethics committee of Tongji Hospital, Tongji

Medical College, Huazhong University of Science and Technology.

\section{Consent for publication}

Not applicable.

\section{Competing interests}

The authors declare no conflict of interest. 


\section{References}

3 1. Hansen K. R., et al. A new model of reproductive aging: the decline in ovarian non-growing follicle number from birth to menopause. Human reproduction. 2008; 23: 699-708.

2. Younis J. S. Ovarian aging: latest thoughts on assessment and management. Curr Opin Obstet Gynecol. 2011 23: 427-34.

3. Quinn M. M., Cedars M. I. Cardiovascular health and ovarian aging. Fertility and sterility. 2018; 110: 790-3.

4. Li L., Wang Z. Ovarian Aging and Osteoporosis. Advances in experimental medicine and biology. 2018; 1086: 199-215.

5. Bethea C. L., Kohama S. G., Reddy A. P., Urbanski H. F. Ovarian steroids regulate gene expression in the dorsal raphe of old female macaques. Neurobiology of aging. 2016; 37: 179-91.

6. Broekmans F. J., Soules M. R., Fauser B. C. Ovarian aging: mechanisms and clinical consequences. Endocrine reviews. 2009; 30: 465-93.

7. Matsuda F., Inoue N., Manabe N., Ohkura S. Follicular growth and atresia in mammalian ovaries: regulation by survival and death of granulosa cells. The Journal of reproduction and development. 2012; 58: 44-50.

8. Turan V., Oktay K. BRCA-related ATM-mediated DNA double-strand break repair and ovarian aging. Human reproduction update. $2020 ; 26: 43-57$.

9. Zhang D., et al. Increased DNA damage and repair deficiency in granulosa cells are associated with ovarian aging in rhesus monkey. Journal of assisted reproduction and genetics. 2015; 32: 1069-78.

10. Jazayeri A., et al. ATM- and cell cycle-dependent regulation of ATR in response to DNA double-strand breaks. Nature cell biology. 2006; 8: 37-45.

11. Morita Y., et al. Oocyte apoptosis is suppressed by disruption of the acid sphingomyelinase gene or by sphingosine-1-phosphate therapy. Nature medicine. 2000; 6: 1109-14. 
1 12. Kaipia A., Hsueh A. J. Regulation of ovarian follicle atresia. Annual review of physiology. 1997; 59: 349-63.

2 13. Hsueh A. J., Billig H., Tsafriri A. Ovarian follicle atresia: a hormonally controlled apoptotic process. Endocrine reviews. 1994; 15: 707-24.

14. Zhou J., Peng X., Mei S. Autophagy in Ovarian Follicular Development and Atresia. International journal of biological sciences. 2019; 15: 726-37.

15. Meng L., et al. Preantral follicular atresia occurs mainly through autophagy, while antral follicles degenerate mostly through apoptosis. Biology of reproduction. 2018; 99: 853-63.

16. Fiscella M., et al. Wip1, a novel human protein phosphatase that is induced in response to ionizing radiation in a p53-dependent manner. Proceedings of the National Academy of Sciences of the United States of America. 1997; 94: 6048-53.

17. Bulavin D. V., et al. Inactivation of the Wip1 phosphatase inhibits mammary tumorigenesis through p38 MAPK-mediated activation of the p16(Ink4a)-p19(Arf) pathway. Nature genetics. 2004; 36: 343-50.

18. Emelyanov A., Bulavin D. V. Wip1 phosphatase in breast cancer. Oncogene. 2015; 34: 4429-38.

19. Yin S., et al. Wip1 suppresses ovarian cancer metastasis through the ATM/AKT/Snail mediated signaling.

20. Demidov O. N., et al. Role of Gadd45a in Wip1-dependent regulation of intestinal tumorigenesis. Cell death and differentiation. 2012; 19: 1761-8.

23. Le Guezennec X., Bulavin D. V. WIP1 phosphatase at the crossroads of cancer and aging. Trends in biochemical 
24. Salminen A., Kaarniranta K. Control of p53 and NF-kappaB signaling by WIP1 and MIF: role in cellular senescence and organismal aging. Cellular signalling. 2011; 23: 747-52.

25. Olcina M. M., Hammond E. M. WIP1 and senescence: oxygen matters. Cell cycle. 2014; $13: 1062$.

26. Sakai H., Fujigaki H., Mazur S. J., Appella E. Wild-type p53-induced phosphatase 1 (Wip1) forestalls cellular premature senescence at physiological oxygen levels by regulating DNA damage response signaling during DNA replication. Cell cycle. 2014; 13: 1015-29.

27. Wong E. S., et al. p38MAPK controls expression of multiple cell cycle inhibitors and islet proliferation with advancing age. Developmental cell. 2009; 17: 142-9.

28. Zhu Y., et al. Phosphatase WIP1 regulates adult neurogenesis and WNT signaling during aging. The Journal of clinical investigation. 2014; 124: 3263-73.

29. Zhou S., et al. The inhibition of WIP1 phosphatase accelerates the depletion of primordial follicles.

30. Wei Y., et al. Integrative Proteomic and Phosphoproteomic Profiling of Testis from Wip1 Phosphatase-

31. Niu P., et al. Male Fertility Potential Molecular Mechanisms Revealed by iTRAQ-Based Quantitative Proteomic Analysis of the Epididymis from Wip1(-/-) Mice. Omics : a journal of integrative biology. 2019; 23: 54-66. arrest in mouse oocytes. Biology of reproduction. 2018; 99: 798-805.

34. Zhou S., et al. Ovarian Dysfunction Induced by Chronic Whole-Body PM2.5 Exposure. Small. 2020; 16. 
35. Zhou S., et al. Ovarian Dysfunction Induced by Chronic Whole-Body PM2.5 Exposure. Small. 2020: e2000845.

36. Shen L., et al. CCL5 secreted by senescent theca-interstitial cells inhibits preantral follicular development via granulosa cellular apoptosis. Journal of cellular physiology. 2019; 234: 22554-64.

37. Shikanov A., Xu M., Woodruff T. K., Shea L. D. A method for ovarian follicle encapsulation and culture in a proteolytically degradable 3 dimensional system. Journal of visualized experiments : JOVE. 2011.

38. Tian Y., et al. Isolation and identification of ovarian theca-interstitial cells and granulose cells of immature female mice. Cell biology international. 2015; 39: 584-90.

39. Yang Y. Q., et al. Wild-type p53-induced phosphatase 1 down-regulation promotes apoptosis by activating the DNA damage-response pathway in amyotrophic lateral sclerosis. Neurobiology of disease. 2020; 134: 104648.

40. Chen Z., et al. Wip1 inhibitor GSK2830371 inhibits neuroblastoma growth by inducing Chk2/p53-mediated apoptosis. Scientific reports. 2016; 6: 38011.

41. Kim J., Kundu M., Viollet B., Guan K. L. AMPK and mTOR regulate autophagy through direct phosphorylation of Ulk1. Nature cell biology. 2011; 13: 132-41.

42. Chen J. H., et al. ATM-mediated PTEN phosphorylation promotes PTEN nuclear translocation and autophagy in response to DNA-damaging agents in cancer cells. Autophagy. 2015; 11: 239-52.

43. Le Guezennec X., et al. Wip1-dependent regulation of autophagy, obesity, and atherosclerosis. Cell metabolism. 2012; 16: 68-80.

44. Brichkina A., Bulavin D. V. WIP-ing out atherosclerosis with autophagy. Autophagy. 2012; 8: $1545-7$.

45. Noda T. Regulation of Autophagy through TORC1 and mTORC1. Biomolecules. $2017 ; 7$.

46. Zhu Y. H., Bulavin D. V. Wip1-dependent signaling pathways in health and diseases. Progress in molecular biology and translational science. 2012; 106: 307-25.

47. Reddy P., et al. Oocyte-specific deletion of Pten causes premature activation of the primordial follicle pool. Science. 2008; 319: 611-3. 
48. Xia Y., Ongusaha P., Lee S. W., Liou Y. C. Loss of Wip1 sensitizes cells to stress- and DNA damage-induced apoptosis. The Journal of biological chemistry. 2009; 284: 17428-37.

49. Lowe J., et al. Regulation of the Wip1 phosphatase and its effects on the stress response. Frontiers in bioscience (Landmark edition). 2012; 17: 1480-98.

50. Uyanik B., Grigorash B. B., Goloudina A. R., Demidov O. N. DNA damage-induced phosphatase Wip1 in regulation of hematopoiesis, immune system and inflammation. Cell death discovery. 2017; 3: 17018.

51. Moskalev A. A., et al. The role of DNA damage and repair in aging through the prism of Koch-like criteria. Ageing research reviews. 2013; 12: 661-84.

52. Hoeijmakers J. H. DNA damage, aging, and cancer. The New England journal of medicine. 2009; 361: $1475-85$.

53. Cha H., et al. Wip1 directly dephosphorylates gamma-H2AX and attenuates the DNA damage response. Cancer research. 2010; 70: 4112-22.

54. Lu X., et al. The type $2 \mathrm{C}$ phosphatase Wip1: an oncogenic regulator of tumor suppressor and DNA damage response pathways. Cancer metastasis reviews. 2008; 27: 123-35.

55. Zhang X., et al. Phosphorylation and degradation of MdmX is inhibited by Wip1 phosphatase in the DNA damage response. Cancer research. 2009; 69: 7960-8.

56. Yoshida K., Miki Y. Role of BRCA1 and BRCA2 as regulators of DNA repair, transcription, and cell cycle in response to DNA damage. Cancer science. 2004; 95: 866-71.

57. Titus S., et al. Impairment of BRCA1-related DNA double-strand break repair leads to ovarian aging in mice and humans. Science translational medicine. 2013; 5: 172ra21.

58. Miao Y., et al. BRCA2 deficiency is a potential driver for human primary ovarian insufficiency. Cell death \& disease. 2019; 10: 474.

59. Shreeram S., et al. Wip1 phosphatase modulates ATM-dependent signaling pathways. Molecular cell. 2006; 23: 757-64. 
60. Gupta S., Silveira D. A., Mombach J. C. M. Towards DNA-damage induced autophagy: A Boolean model of p53induced cell fate mechanisms. DNA repair. 2020; 96: 102971.

61. Chen Z., et al. Wip1 deficiency impairs haematopoietic stem cell function via p53 and mTORC1 pathways. Nature communications. 2015; 6: 6808.

5 62. Yi W., et al. Phosphatase Wip1 controls antigen-independent B-cell development in a p53-dependent manner. Blood. 2015; 126: 620-8. 
2 Fig.1 The expression of WIP1 in mouse ovaries. (A) Representative images of WIP1 expression in different stages follicles in the mouse ovaries by IHC. (B) Relative WIP1 expression according to mean density (IOD/area). (PMF: primordial follicles, PF: primary follicles, SF: secondary follicles, ANF: antral follicles, THF: total healthy follicles, ATF: atretic follicles, CL: corpus luteum) Data are presented as the mean (SD). * $p<0.05$. experiment. (B) Body weight during the gavage administration. (C) The proportion of regular estrous cycles. (D) The proportion of pregnant mice of total mated mice. (E) Average litter size of total mated mice. (F) Ovary index (ovary weight/body weight). (G) Serum AMH level. (H) The representative HE staining images of mouse ovaries. (I) Follicle counting results according to ovary serial sections $(n \geq 4)$. (J) The proportion of follicles at different stages $(\mathrm{n} \geq 4)$. Data are presented as the mean (SD). ${ }^{*} p<0.05$.

Fig.3 The granulosa cell apoptosis contributed to the follicular atresia after treated with WIP1 inhibitor. (A) TUNEL staining of mouse ovaries with or without GSK2830371 treatment. (B) Relative TUNEL expression ( $\mathrm{n} \geq 3$ ). (C-D) Protein expression of DNA damage and apoptosis related genes detected by Western blot. Data are presented as the mean (SD). ${ }^{*} p<0.05$.

Fig.4 The autophagy participated in the follicular atresia after treated with WIP1 inhibitor. (A) Representative images of LC3B staining in mouse ovaries. (B) Relative LC3B expression based on IOD scores. (C) Representative images of SQSTM1 staining in mouse ovaries. (D) Relative SQSTM1 expression based on IOD scores. (E-F) Protein expression of autophagy related genes detected by Western blot. Data are presented as the mean (SD). ${ }^{*} p<0.05$. 
Fig.5 The effect of WIP1 inhibitor on secondary follicle development invitro. (A) The morphological analysis of follicle growth. (B) Diameters of follicles treated with different concentrations of GSK2830371. (C) Ki67 staining

follicles. (E) Microphotographs showing the in vitro maturation of follicular oocytes. (F) The percentages of the oocytes in each stage were quantified. (GV, germinal vesicle; GVBD, germinal vesicle breakdown; MII, metaphase II) Data are presented as the mean (SD). * $p<0.05$.

8

Fig.6 The effect of WIP1 on primary granulosa cells cultured in vitro. (A) The morphology of mouse granulosa cells. (B) Granulosa cell proliferation detected by EdU. (C) Granulosa cell apoptosis detected by FACS. (D) Granulosa cell apoptosis detected by TUNEL. (E-F) Protein expression of autophagy and apoptosis related genes detected by Western blot. Data are presented as the mean (SD). * $p<0.05$.

\section{Fig.1}
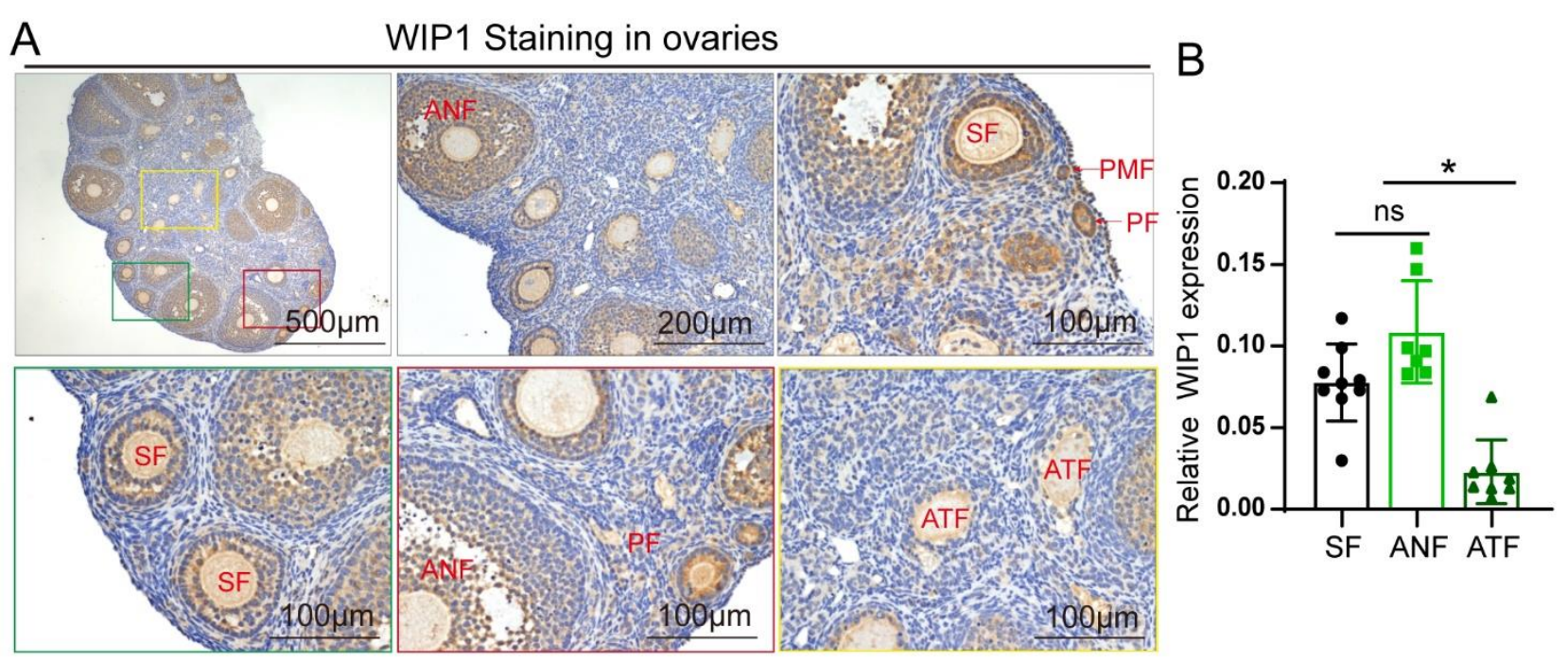
Fig.2

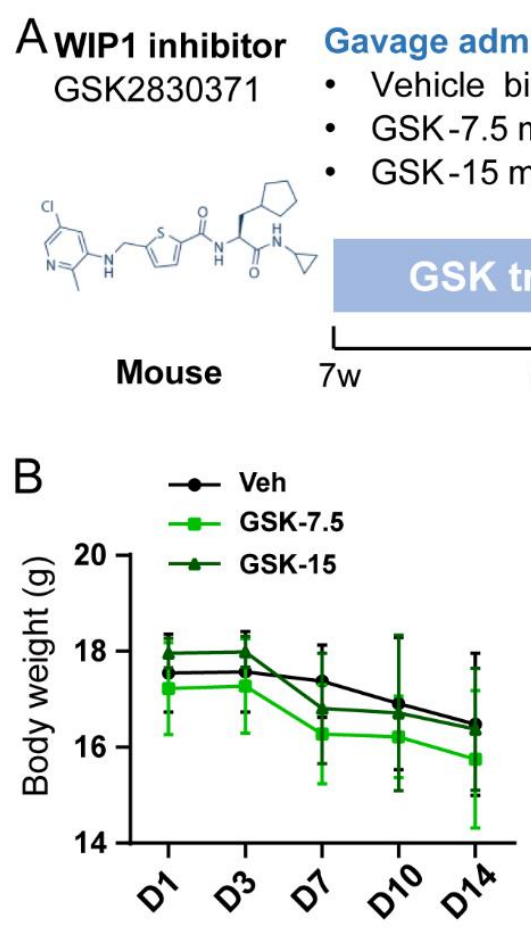

\section{Ovary}

Morphological analysis

Molecular analysis
F

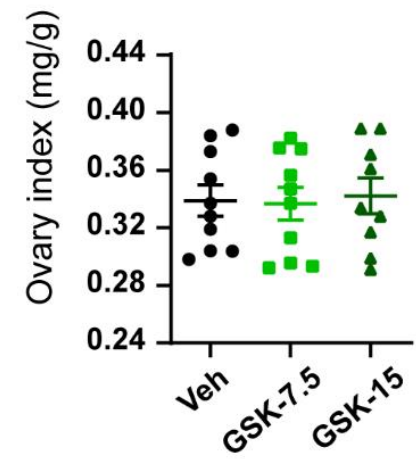

I
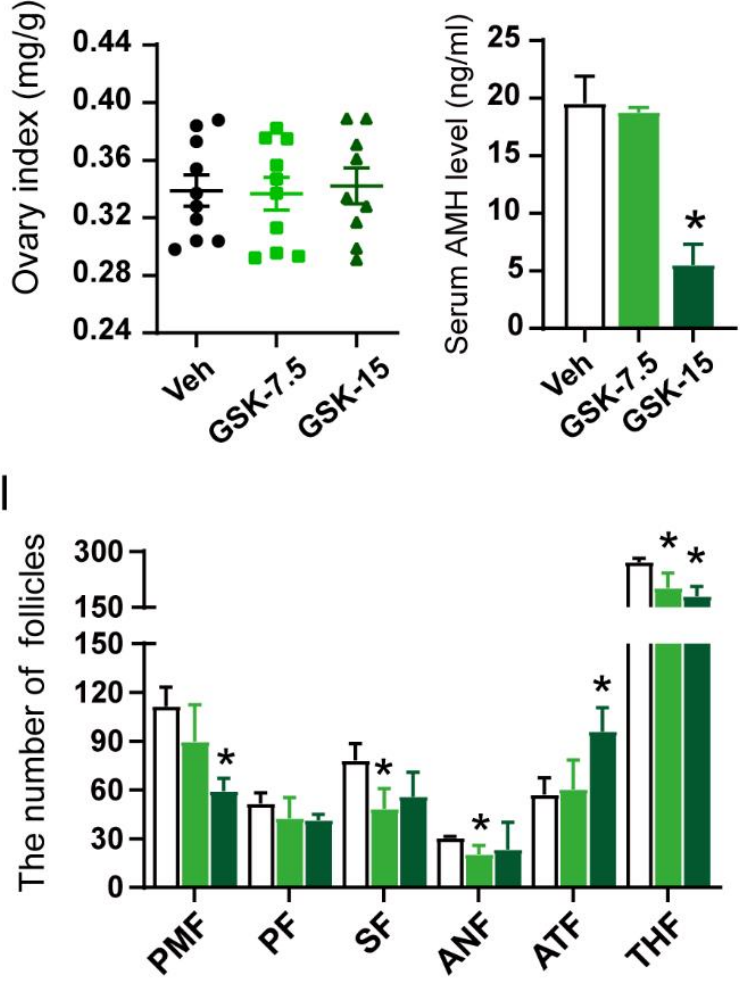

Mating test

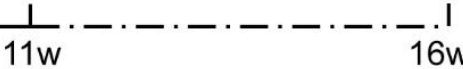

$E$
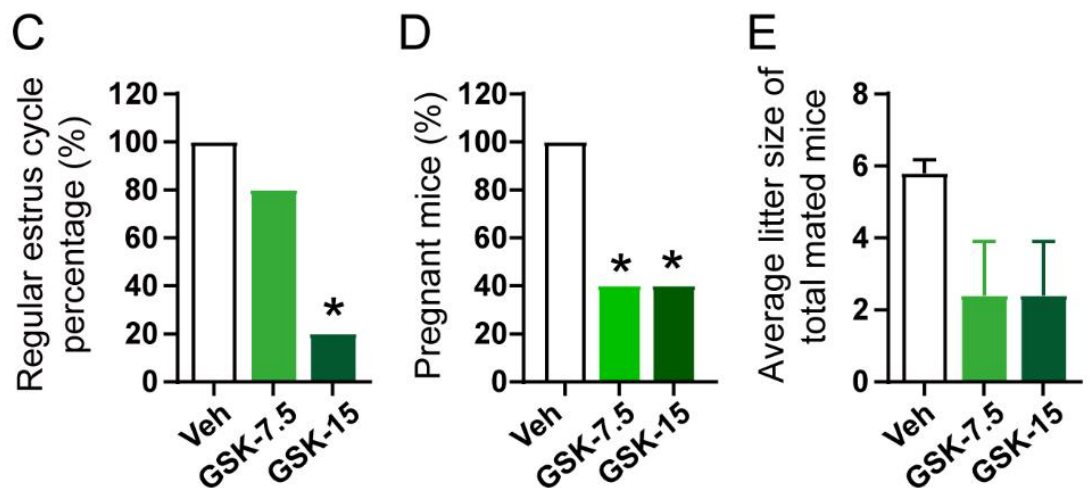

$\mathrm{H}$

H\&E Staining

宛
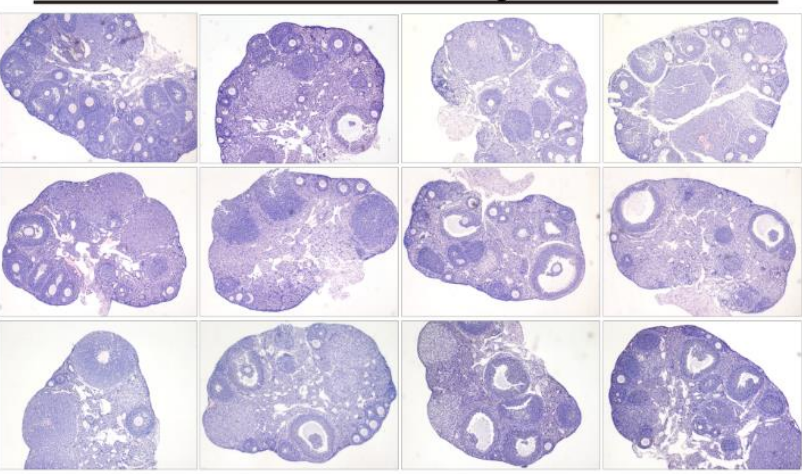

$\mathrm{J}$

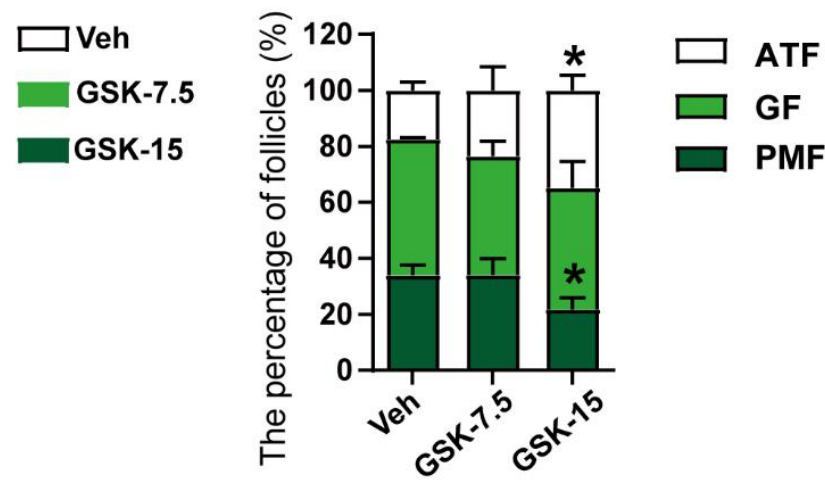


$1 \quad$ Fig.3

A

Hochest

TUNEL

TUNEL
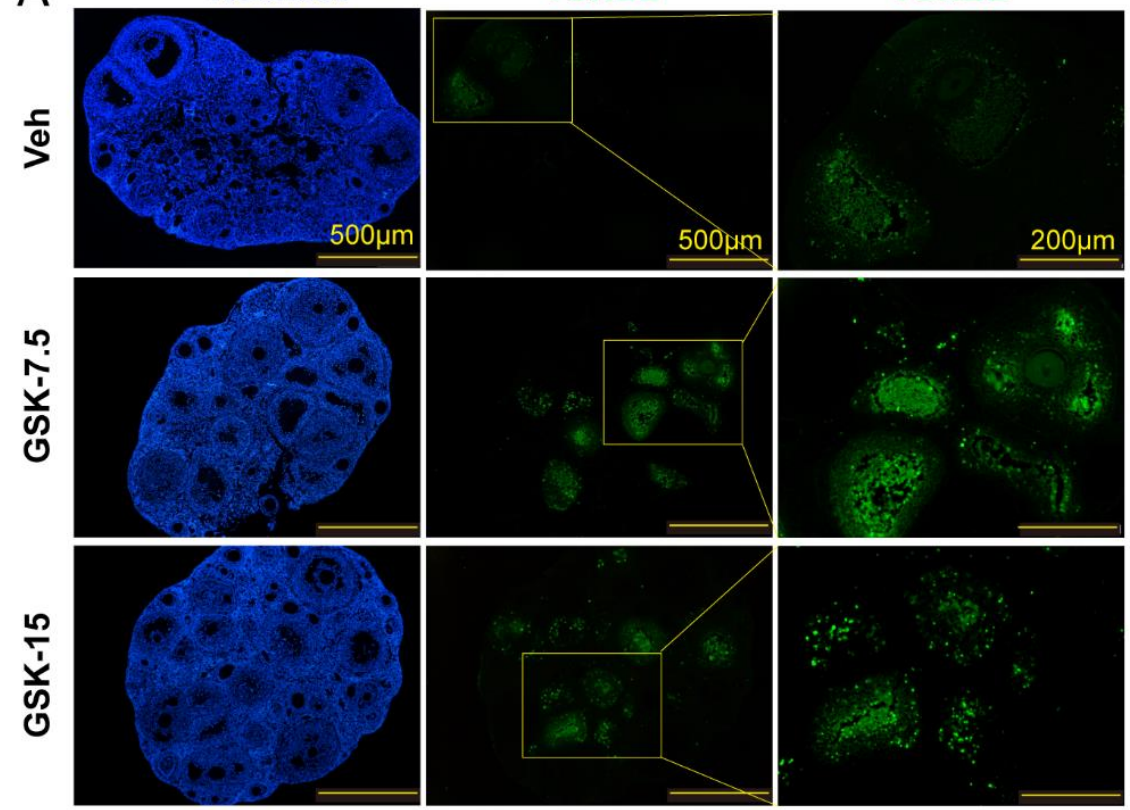

B

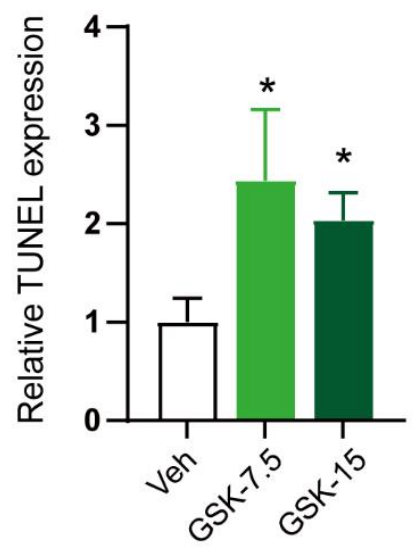

C

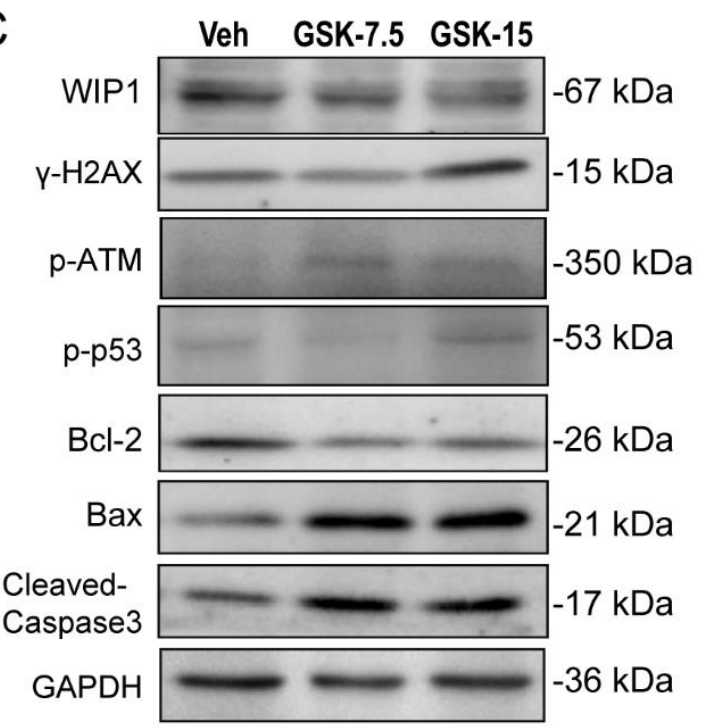

D

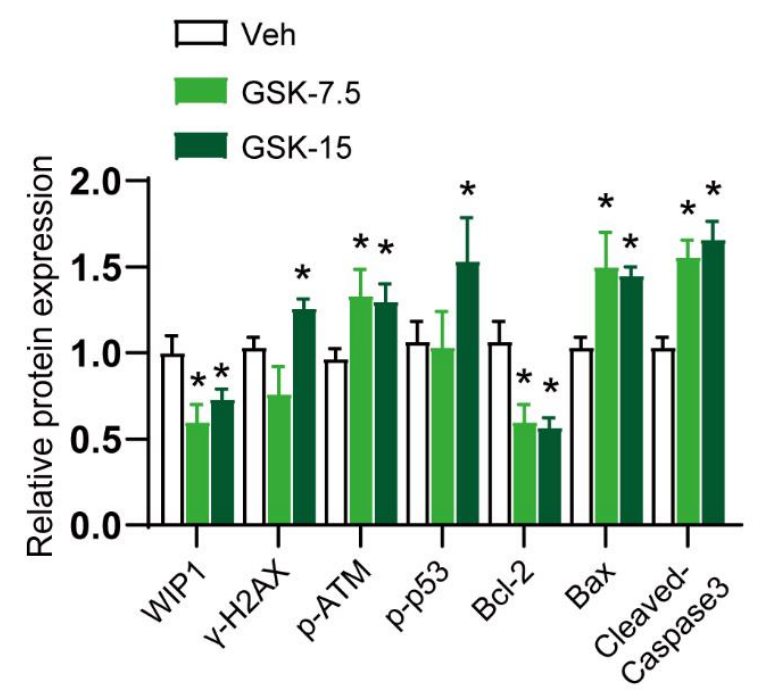


Fig.4

A

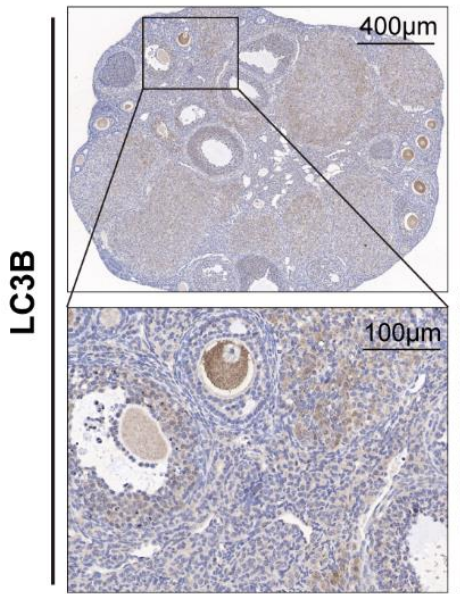

C

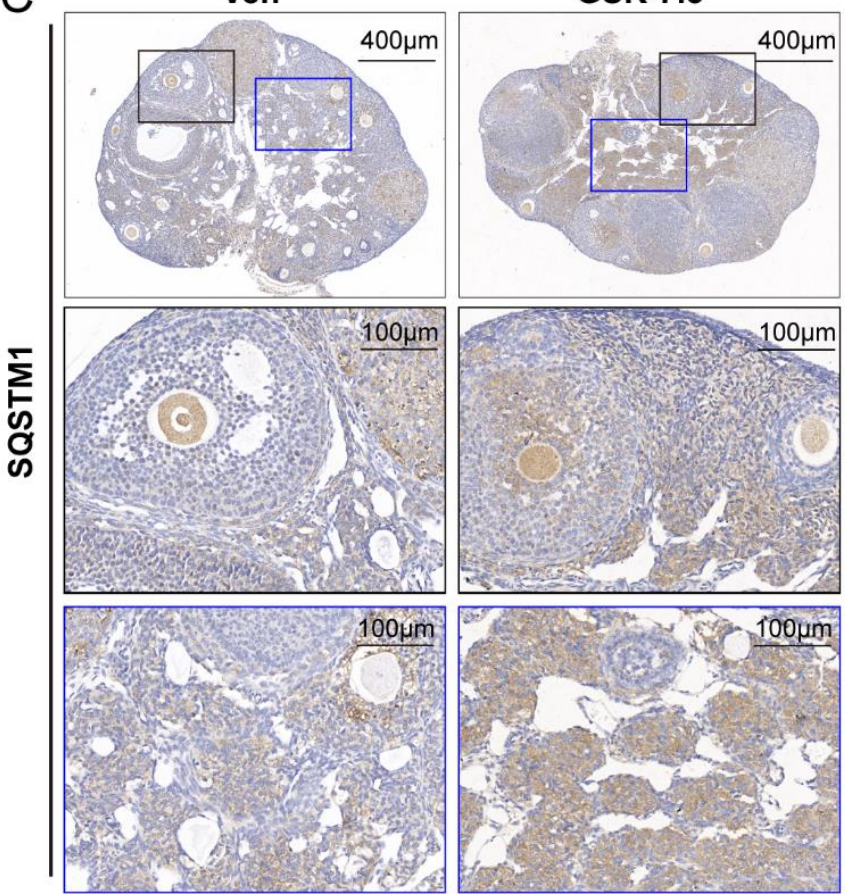

E

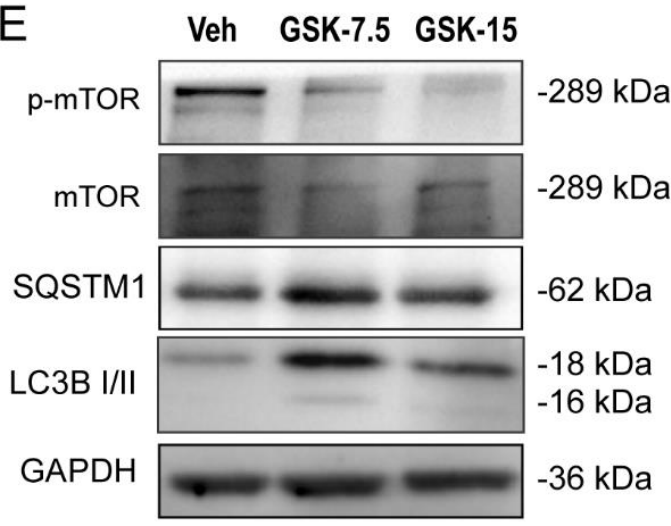

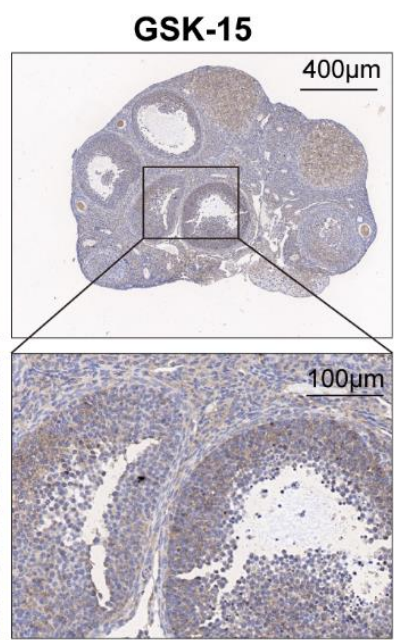

B

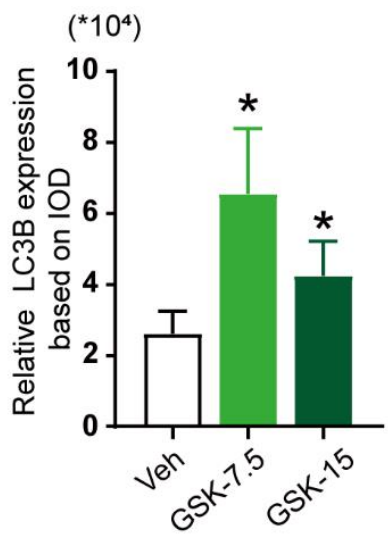

GSK-15

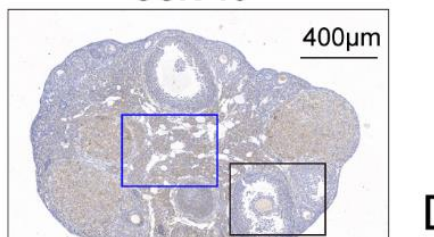

D

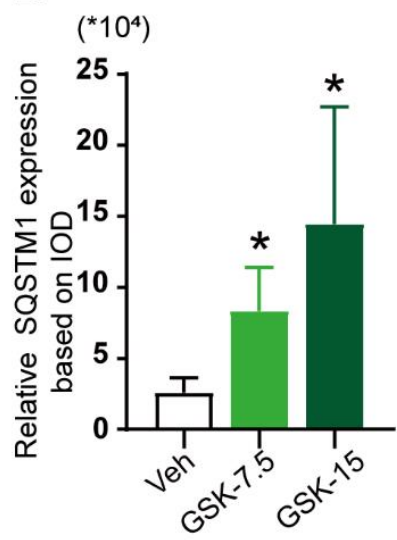


Fig.5
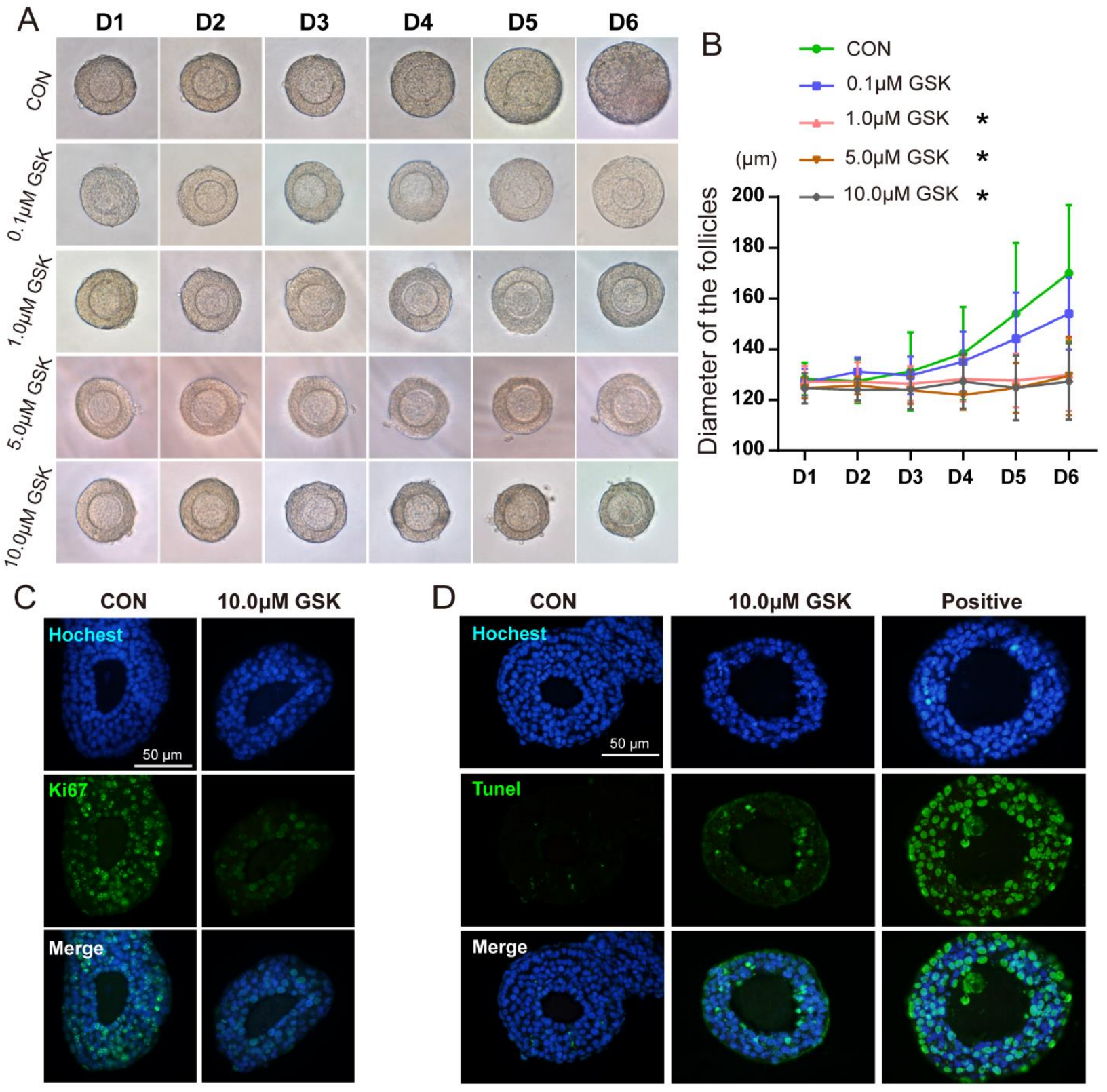

E
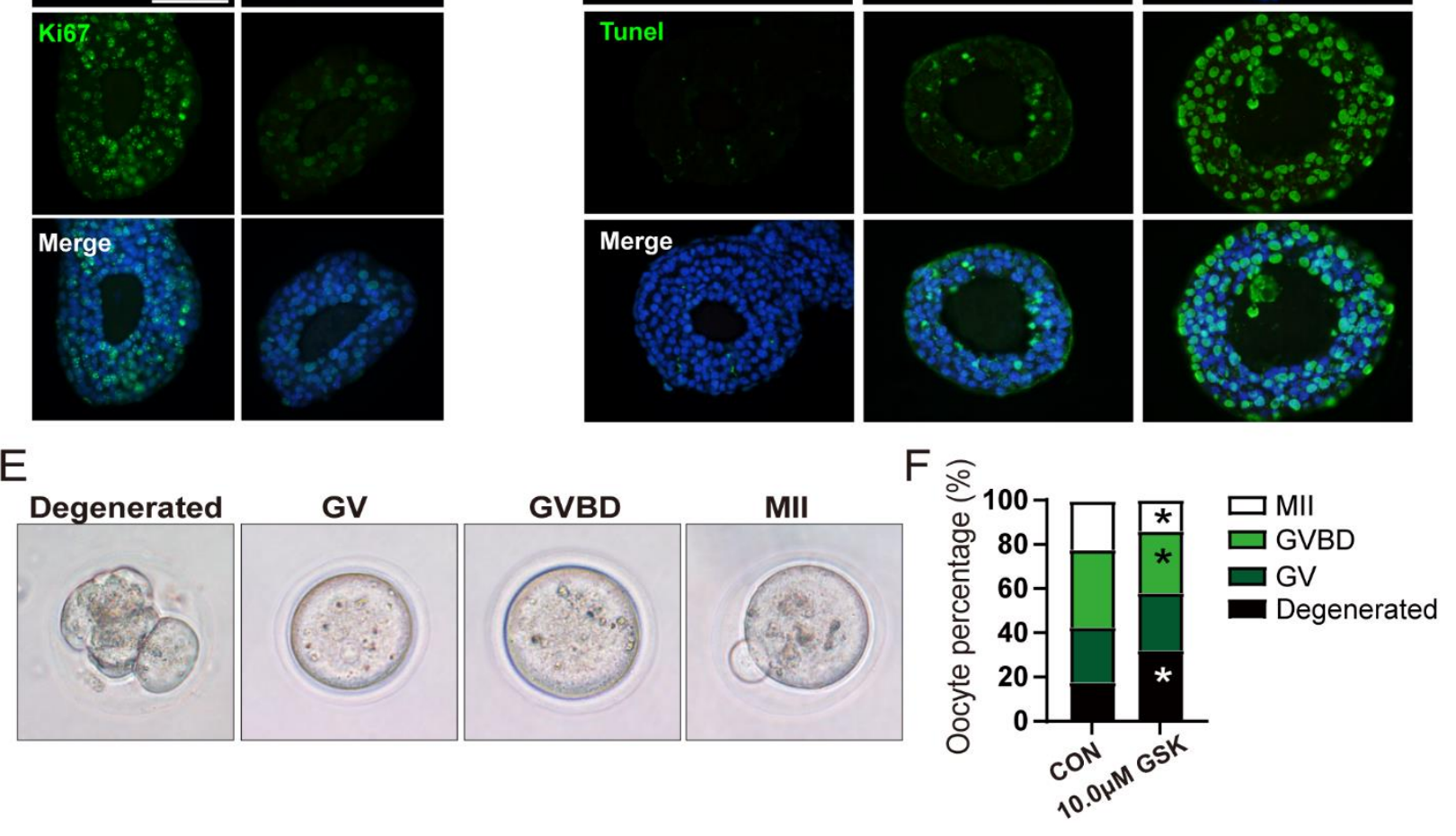
Fig.6
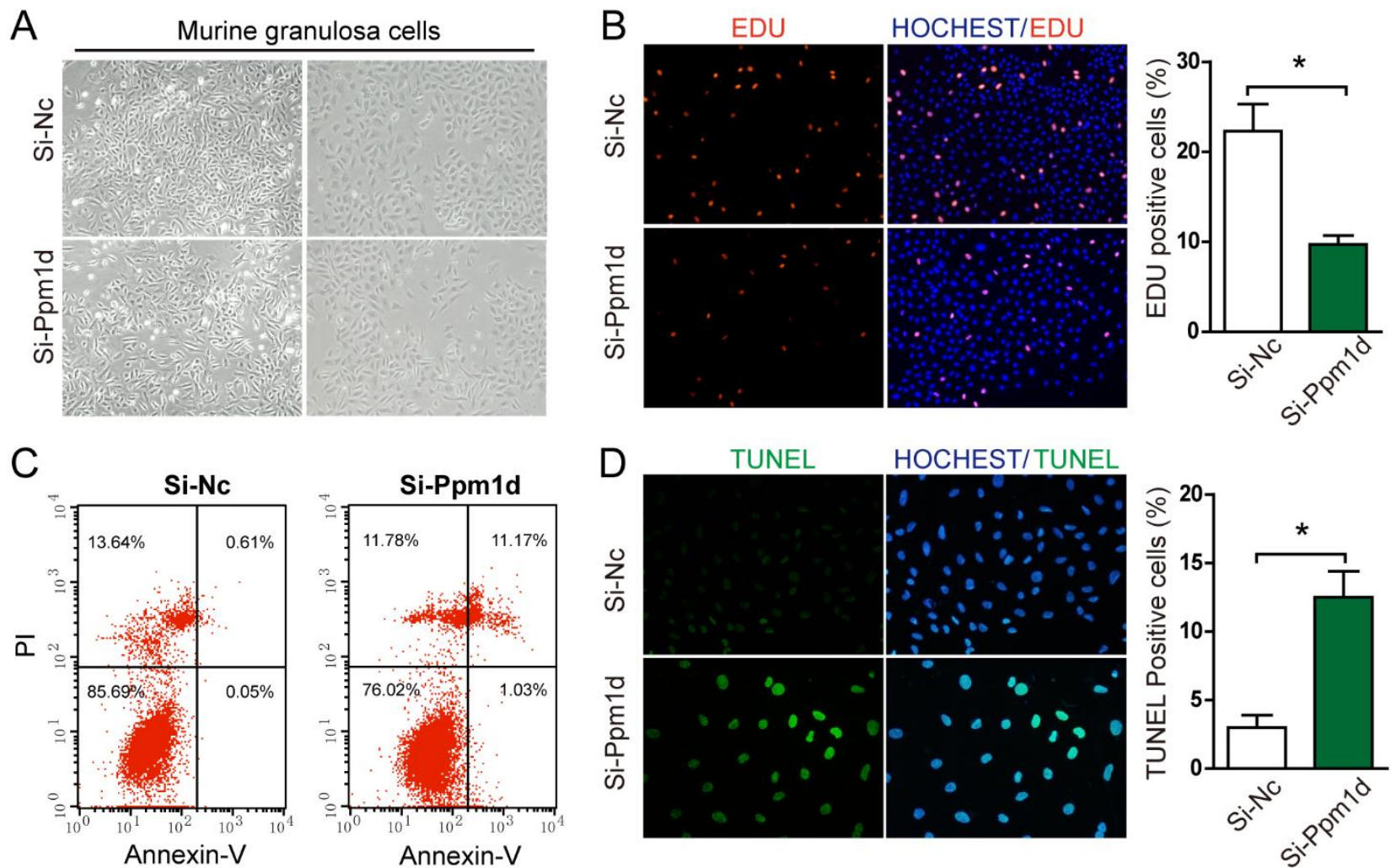

E

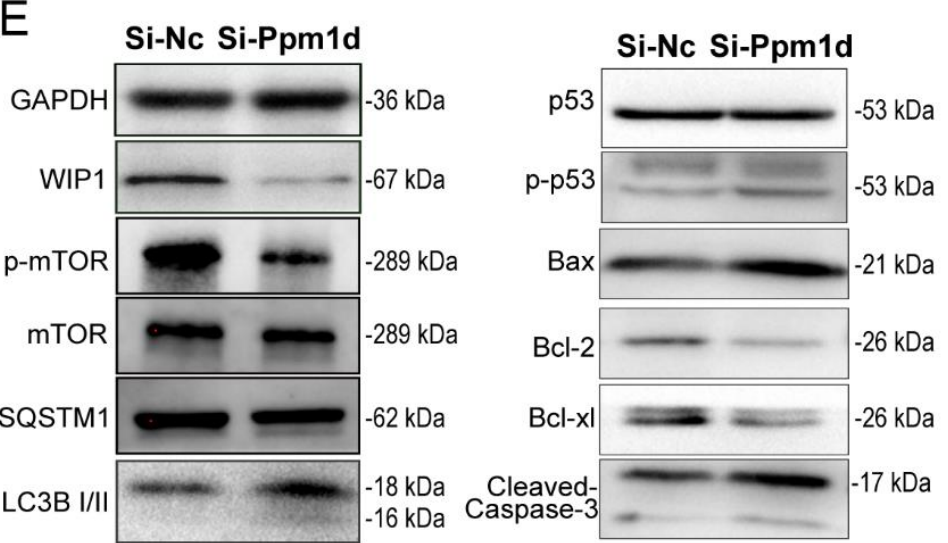

F $\square \mathrm{Si}-\mathrm{NC}$

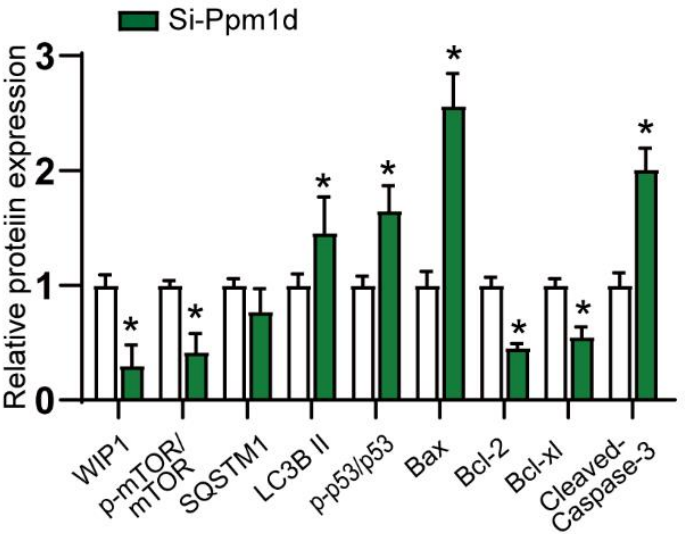

